\title{
Karl Korsch Jus belli ac pacis im Arbeitsrecht
}

An der Sçwelle der modernen bürgerlichen Rechtswissenschaft steht das epochemaciende Werk des großen Theologen, Philosophen und Juristen Hugo Grotius: "De jure belli et pacis libri tres« (Drei Bücher über das Recht des Krieges und Friedens). Der gelehrte und berühmte Verfasser nahm se!bst ahtiv teil an jenen langwierigen und häufig blutigen Kämpfen, in denen damals in aller Ländern des alten und des neven Occidents die aufsteigende bürgerliche Klasse die Welt gewalisam nach ihrem Bilde umgestaltetc. Er wurde dafür von den Niederländischen Generalstaten auf Befehl des Prinzen Moritz von Oranien im Jahre 1618 als Hochverräter verhafter und in den Kerker geworfen, aus dem er nach einigen qualvollen Jahren mit Hilfe seiner treuen Gattin entwich, um dann die leezten Jahrsehnte seines Lebens im glänzenden Elend des Exils zu verbringen. Sein Werk aber blieb für Jahrzehnte ein Hauperwerk der juristischen Wissenschaft, - solange, bis mit dem allmählichen Verfall der bürgerlicinen Rechrswissenschaft im 19. Jahrhundert das gesamte Naturrecht und mit ihm auci die Lehre des Grotius als ein wissenschaftlich überwundener Standpunkt angesehen wurde, der für die erleschtete Gegenwart nur noch historische Bedeutung hätze. An die Stelle des wahrhât w wissenschaftlicien «, lebendigen, schöpferischen und revolutionären Geistes der Naturrechrslehrer trat in dieser Epoche, in der die bürgerliche Ǩlasse im westlichen und mitrleren Europa allmählich aufhörte, eine revolutionäre Klasse, ein sich emanzipierender und um die Suprematie in Staar und Gesellsciaft kämpfender »dritter Stand " zu sein, ein verdrießliches, unfrucitbares und gänzlich unwissenschafliches Epigonentum, welches seine eigene Leerheir und Scrwäne, seinen Historismus und Positivismus als der Rechisweisheit lezzten und sichersten Schluß hinstellte. Der Ritte: Hugo*, der sich zu dem von inm immerfort angerufenen Philosophen Kant so verhält, wie der Afe zum Menschen, erschilug mit seinem $*$ Naturrecht $\alpha$, dem von Karl Marx gegeilelten "Míanifest de: historischen Rechessciule « das Naturrecht des Hugo Grotius, und die moderne Rechtswissenschat ve:fiel, zumal in Deutschland, jener $n$ wissenschaftlichen Wertlosigheir «, die ihr um das Jahr I $\$ 48$ der philosophierende Jurise Kirchmann, von seinem Standpunkt aus mit Grund, a!s einen allgemeinen Charakter attestierte. Der innerc Wesenskern des Recirs war für diese neue und moderne, historische und positive, bürgerliche Rechtswissenschaft unbegreiflich geworden. Sie nahm mit wenig Witz und viel Behagen die Schale für den Kern, das Wort und sein Begriff trat an die Stelle des Begriffs der Sache, und seibst der Begriff des Begriffs vom Recht ging so verloren.

Eine große Menge von wissenschaftlichen Einsichten in das wahre Wesen des

* Anm. d. Red.: Gustzy Hugo, 1764-1844, Begründer der historischen Rechrssconvie; $1729 \mathrm{cr}$ sciien sein sLchrbuch des Naturecitese; vgl. hierzu auch Ernst Block, Naturrecite und mensct:licie Würde, subrkamp taschenbuci Nr. 49, Fim. 1972, S. 103. 
Rechts, die das ältere und neuere Naturrecht bereits besessen hatten, gerieten in dieser Verfallsperiode der bürgerlichen Rechrswissenschatt in völlige Vergessenheit. Sie wurden von dem modernen juristischen Historismus und Positivismus verständnislos und bedenkenlos weggeworfen. Zu den größten Verlusten, die auf diese Weise entstanden sind, gehört der Verlust der Einsicht des Grotius, daß alles Recht, seinem wahren Begriff nach betrachtet, ein Recht des Krieges und des Friedens ist. Diese Einsicht des Grotius, die während der ganzen naturrechtlichen Epoche in der einen oder anderen Form festgehalten worden ist, bedeutet etwas ganz anderes als die moderne Einteilung des sogenannten Völkerrechts in zwei Teile, das Kriegsreche und das Friedensrechr. Krieg und Frieden bilden nach Grotius ein gesellschaftliches Ganzes, das als solches Ganzes auch vom Rechesgelehrten begriffen und weiter entwickelt werden muß. Die Epigonen zerteilten und zerstörten dieses lebendige Ganze des Rechts. Sie lösten das Recht des Krieges a als einen besonderen, übrigens nicht einmal ganz legitimen «, in seinem Rechtscharakter nicht ganz unanfechtbaren, Teil des Rechtssystems von dem lebendigen Gesamtkörper ab und setzten es selbständig für sich. So wurde der Krieg für sie ein Gebier, für welches das sonst so genannte »Recht«, das normale, ganz und gar positive Recht des Friedens, keine Geltung mehr hatte und an seine Stelle ein besonderes, nicht mehr ganz normales und nur noch sehr notdürftig positives "Kriegsrecht« trat, welches überdies, wie die modernen Kriege zeigten, die wirklichen Zwedre der kriegführenden Menschen größtenteils mehr störte und behinderte als wahrhatt förderte, während doch das eigentiche, normale, für die Friedensverhältnisse geltende Recht den friedlichen Handelsverkehr und alle sonstigen Geschäfte des Friedens trägt und fördert.

Zwei ungeheure Erscheinungen der gegenwärtigen Weltgeschichte sind geeignet, den falschen Schleier zu zerreißen, den diese abstrakte und oberflächliche Rechtsauffassung der modernen bürgerlichen Juristen über das wahre Wesen des Reches ausgebreitet hat. Die eine dieser Erscheinungen wird gebildet durch die furchtbare Tatsache des Weltkrieges 1914-1918 und seine bis in die Gegenwart und Zukunft fortwirkenden Folgen. Die andere besteht in den seit Beginn des Jahrhunderts an Breite, Tiefe und Schärfe ständig zunehmenden wirischaftlichen Kämpfen $z$ wischen den sogenannten Arbeitgebern und Arbeitnehmern.

Der große Weltkrieg hat jedem, der Augen hat zu sehen und Ohren zu hören, deurlich und offenbar gezeigt, $\dot{d} a ß$ in der modernen bürgerlichen Gesellschaft der Krieg, als aktuelles oder potentielles Moment, allgegenwärtig ist, und das dieser allgegenwärtige Krieg in der modernen bürgerlichen Gesellschaf, im großen betracintet, die Geschäfte der Bürgcr dieser Gesellschaft auch durchaus nicht stört und behindert, sondern vielmenr als die unerläßliche Vorbedingung, als der mächrigste Hebel und Förderer aller wirklich lohnenden Geschäfte innerhalb dieser Gesellschaft zu gelten hat. So ergibe sich für eine Recheswissensciaft, die das Leben begreif, der SchluB, das auch noch heute, im vollen Iichte unserer kultivierten und humanisierten modernen bürgerlichen Gesellschaft das Recht dieser Gesellschaft, das heißr das gesamte privaze und öffentiche bürgerliche Recht nur als Recht des Krieges und des Friedens begrifien werden kann, ganz ebenso, wie es von Hugo Grotius und seinen revolutionären bürgerlichen Zeitgenossen vor 400 Jahren und von ihren Nachfolgern, den großen Naturrechtslehrern bis zu Kant, Fichte und Hegel begriffen worden ist. Wir haben auch in den letzten Jahren sehen können, daß ein Teil der jüngeren Generation der Juristen ein gewisses Bewußrsein davon erlangt hat, daß eine Jurisprudenz, die alles Recht, von einem bestimmten abgesonderten Teil des Völkerrecits abgesehen, wesentlich als $\times$ Friedensordnung * begreifen will, für die gegenwärtigen 
Bedürfnisse niche mehr ausreicht. Weit entfernt sind aber auch noch diese, in den leszten Jahren an ihrem früheren Glauben und Denken irregewordenen bürgerlichen Juristen von einern wirklichen Begreifen der Tatsache, daß allem und jedem rechrlichen Geschehen innerhalb der heutigen bürgerlichen Gesellschaft, dem privatesten Rechtsverkehr ganz cbenso wie den imperialistischen Welthändeln, ein kriegerisches Moment innewohnt, und noch weniger vermögen sie zu begreifen und einzusehen, worin denn dieses allem rechtichen Geschehen einverleibre kriegerische Moment konkret und wirklich besteht. Diese Unfähigkeit, etwas wirklich Vorhandenes zu sehen und zu begreifen, hat ihre letzte Ursache darin, daß dic bürgerliche Recheswissenschaft vermögc ihres besonderen geschichtlichen und gesellschaftlichen Charakters gewisse Wirklichkciten und Wirklichkeitszusammenhänge nicht sehen und begreifen dari und kann, durch deren Begreifen und Erkennen sie aufhören würde, das zu sein, was sie allen ihren Bekenntnissen zu dem Geist freier voraussetzungsloser wissenschaftlicher Forschung zum Trotz, oder vielmehr auch gerade mit diesen ihren Bekenntnissen, in ihrer geschichtlichen Wirklichkeit tatsächlich ist, nämlich eine bürgerliche Klassenwissenschaft, ein Teil der Wissenschaft der bïrgerlichen Klasse.

Um dies zu zeigen und zu beweisen, wenden wir uns sogleich zu demjerigen $\mathrm{Ge}-$ biets des Rechts, dem der Charakter eines Kriegs- und Friedensrechts am allerunmittelbarsten und deshalb auch am allersichtbarsten aufgeprägt ist, dessen wirkliche Erscheinungen durch eine von dcm Gedanken des Krieges abstrahierende Betrachtung am allerwenigsten begrifien werden können, und welches merkwürdigerweise doch zugleich dasjenige Gebiet ist, für welthes dieser Gesichrspunkt von der gesamten bürgerlichen Recheswissenschaft unscrer Zeit und von ihren getreuen Trabanten, der Rechtswissenschaft des modernen sozialdemokratischen Reformismus d la Sinzheimer und Flatow, am allerschärfsten zurüdgerviesen wird. Wir meinen das Rechtsgebiet, welches von dem berühmten Artikel i 57 der Weimarer Verfassung und von der heurigen Rechtswissenscriaft als das "A-beitsrecht» bezeichnet wird. Gerade an diesem Arbeitsreche läßt sich der $z$ wingendste Bewweis dafür erbringen, daß die heutige bürgerliche Rechtswissenschaft ganz ebenso wie die heutige bürgerliche Philosophie und die heutige bürgerliche Okonomie den wirklicien Erscheinungen der gegenwärtigen Entwicklung nicht mehr unbefangen, kritisch und ohne unbewußte Voraussetzungen, das heift genaver gesprochen, nicht mehr ohne Klassenvorurteile gegenübersteht und darum das wahre Wesen dicser Erscheinungen nicht mehr richrig und vollständig zu begreifen vermag. Ganz ebenso wie auf dem Gebiet der Philosophie und der Okonomic, so sind auch auf dem Gebiete der Rechtswissensciaft die in ihrer gese!lschaftlichen Praxis gegenrevolutionär gewordenen Gelehrten der bürgerlichen Verfallsperiode auch in ihrer wissenschaftlichen Theorie nicht mehr dazu befähigt, jene wahrhaft wissenschaftlicien Methoden der E-kenntnis anzuwenden und wciter zu entwickcln, die ihre wissenschaftlichen Vorfahren, die alten und neuen Naturrechtslehrer der bürgerlichen Revolutionsperiode, in die Rechtswissenschaft eingefünrt haben. Auch in der Rechtswissenschaft, wie in der Philosophie und Okonomie, ertweist sich als die wirkliche E-bin der klassischen deutschen Geistesberiegung nicht die of fizielle bürgerliche Wissenschaft die dem rüd:sichtslosen kritischen Geiste der Klassiker verständnislos gegenübersteht, sondern vielmehr die deutsche und die internationale Arbeiterbewegung, die an die philosophischen und wissenschaftlichen Traditionen der bürgerlichen revolutionären Naturrechtscpoche anknüpft und aus dieser Anknüpfung heraus ihre neue proletarische revolutionäre Mcthode der Rechtserkenntnis und Rechtsgestaltung entwidkelt. 
Das Arbeitsrecht ist das Recht des Friedens und des Krieges für die beiden in der gegenwärtigen bürgerlichen Gesellschaft miteinander kämpfenden großen Klassen, die herrschende Bourgeoisie und das sich gegen diese Herrschatt empörende Proletariat. Wer an diescr Erkenntnis vorbeizugehen, sie zu umgehen oder zu bestrciten versucht, vermag überhaupt nicht zu erklären, warum es denn notwendig sei, das übliche und längst eingebürgerte System der Rechtwissenschaft gerade an dieser Stelle so unlogisch zu durchbrechen, und die verschiedenartigsten Bestimmungen des bürgerlichen Privatrechts und des bürgerlichen öffentlichen Rechts, das Handels-, Gewerbe-, Verwaltungsrecht, des matericllen und prozessualen Rechts aus ihrem wnatürlichen systematischen Zusammenhang herauszurcißen und zu einem besonderen Block, dem des sogenannten »Arbcitsrechts zusammenzufügen. Die langatmigen und gekünstelten Begründungen, mit denen die bürgerlichen Juristen dieses durch den Begriff und den Namen des *Arbeitsrechts * aufgegebene Problem zu lösen versucht haben und noch versuchen, können nicht die Tatsachen hinwegräumen, daß es keinem Rechtsgelehrten jemals eingefallen wäre, dem Arbeitsrecht eine begriffliche Selbständigkeit zu vindizieren, wenn nidit die Realität der proletarischen Klassenkämpfe, vor allem der großen wirtschaftichen Arbeitskämpfe (Streiks und Aussperrungen) in allen Ländern, dazu immer und immer wieder einen triftigen Realgrund dargeboten hätte. Es läßt sić weiterhin sehr leicht zeigen, daß ein großer, sogar der größte und wesentlichste Teil dessen, was man heute selbst als streng bürgerlicher Jurist unter dem sogenannten *Arbeitsrecht « versteht, überhaupe gar kein bürgerliches Recht mehr ist und weder unter das sogen. Privatrecht noch unter das sogen. öffentliche Recht der heutigen bürgerlichen Gesellschaft ohne Zwang subsumiert werden kann. Manche haben es darum "Sozialrecht» genannt, ohne über die Gründe dieser neuen Terminologie sich selbst und uns genügend klare Rechenschaft zu geben. Sie haben nur ein neues Wort geprägr, nach dem Goetheschen Spruch, daß eben wo Begriffe fehlen, da stellt ein Wort zur rechten Zeir sich ein. Daß sie den zu dem Wort gehörigen neuen Begriff der neuen Sache tatsächlich nicht haben, beweisen sie dadurch, daß sie dies ihr soziales Recht ganz ebenso frisch und munter als eine, mutatis mutandis gleichartige, dritte Gruppe von bürgerlichen Rechtsnormen neben dem bürgeriicien Privatrecht und dem bürgerlichen öfrentlichen Recht in das bürgerliche Rechtssystem hineinstellen wollen. Tatsächlich aber handelt es sicn bei dem sozialen Arbeitsrecht in keinem Sinne mehr um etwas, was dem normaien privaten oder öffentlichem Reche der bürgerlichen Gesellschaft wesensgleich wäre. Die meisten Arbeitskämpfe, denken Sie z. B. an einen großen Streik, der ausgeführt wird von Arbeitern, die nach ihrem privaten Arbeitsvertrag auf jederzeitige fristose Kündigung eingestell s sind, ein solcher Arbeitskampf, der z. B. die gewaltsame Herbeiführung einer Arbeitsverkürzung oder einer Lohnerhöhung bezwedst, wird überhaupt nicht geführt um irgendwelche, sei es privare, sei es öffentliche existierende Rechte von Einzelpersonen (und das bürgerliche Recht kennt doch nur solche Rechte von Einzelpersonen, natürlichen und juristischen Personen!). Ewird vielmehr geführt als eine sogen. Gesamtstreitigkeit «, als ein kollektiver Streit um Rechte, die für eine privatrechtlich unbestimmte und mit privatrechtlichen Mitteln nicht bestimmbare Personenmehrheit durch den erfolgreichen Kampf des Streiks erst geschaffen werden sollen. Er ähnelt also, schon rein äußerlich betrachtct, weit mehr einem Krieg zwischen seibständigen Staaten, der in einem Friedensschluß endigt als einem bürgerlichen Streit von Privatpersonen oder auch von innerstaatichen Behörden um bestimmte, rechtich geregelte Verhältnisse. Und wo steht es geschrieben, daß ein „Kriega nur zwischen souverä- 
nen Staaten möglich sei und nicht auch $z$ wischen ganz andersartigen kollektiven Gebilden? Auch hier, bei der Definition des Krieges zwischen Staaten und Völkcrn, haben wir es durchaus nicht mit ciner natürlichen und selbstverständlichen, immer so gewesenen Begriffsbestimmung zu tun, sondern mit einer eigens für den Gebrauch der modernen, von der bürgerlichen Klasse beherrschten Gesellschaft, geschaffenen $*$ wissenschaftlichen $\alpha$ Begriffsbestimmung ndes* Krieges. Hugo Grotius wußte von dieser künstlichen Einschränkung noch nichts, und mit überlegener Miene haben ihn die Epigonen des I9. Jahrhunderts darob getadelt, daß er *mehrere Arten des Kricges« unterschieden hat, neben dem öfentlichen Krieg noch den privaten Krieg, und innerhalb der ersteren Art wieder verschiedene Arten, von denen nur eine die Krieg $\mathrm{z}$ wischen den Staaten ist. Der Krieg bezeichnet, nach des Grotius Definition, *den Zustand von Personen, die miteinander gervaltsam kämpfen « (Buch I. Kap. I). Und ebenso lehrt noch der größre Kriegsohilosoph, den die bürgerliche Klasse am Ausgang ihrer revolutionären Epoche hervorgebracht hat, der berühmte General Karl von Clausewitz in seinen acht Büchern „Vom Kriege «, der Kriez sei, „ein Konflikt großer Interessen, der siá blutig löst, und nur darin sei er von den andern (Konflikten) verschieden $\propto$; und auch dieses letztere Kriterium, die Notwendigkeit der ×blutigen « Iösung, hält er nicht als unentbehrliches Merkmal des Krieges fest, sondern läßt in der Folge auch solche $*$ Kriegex noch begrifflich zu, die * in bloßer Bedrohung des Gegners und in einem Subsidium des Unterhandelns bestehen" (III, I;8). Nichts stehe also im Wege, daß wir diese nachträglicine Begriffsverengung der Epigonen wieder aufheben, und auch die wirsschaftlichen, sozialen und politischen Klassenkämpfe der proletarischen Klasse gegen die ökonomisch, sozial und politisch herrsciende bourgeoise Klasse nicht nur als "Kämpfe» bezeichnen, sondern in ihnen die direkten *Kriege" erkennen, die sie ih:em. Begriffe nach selbst in ihren unentwidkeltsten und unscheinbarsten Formen bereits sind, und zu denen sie sich, wie wir gleich noch sehen werden, letzten Endes auch in ihrer wirklicien geschichtlicien Eascheinung entwickeln und steigern.

Mit dieser Einführung des Kriegsbegrifies in die neue Wissenschaft des heutigen Arbeitsrecint ist wissenschaftlich weit mehr gewonnen als bloß ein neues Wort. Wir gewinnen mit einem Schlage das geistige Band, das die ganze, scheinbar so disparate und heterogene, Masse der heurigen arbeitsrecitilichen Normen zu einer festen Einheit zusammenschließt. Alle unseren. Vorsteilungen über arbeitsrechuliche Verhälenisse bekommen scion hierdurch einen schär:eren Sinn, eine festere Ricintung und eine nähere Anwendung. Darüber hinaus wrird es uns durch dieses neue Prinzip möglich, nicht nur das gegenwärrige Arbeitszecht in ailen seinen Erscheinungen, sondern aucì seine geschichtliche Entwidklung, in der Vergangenheit und ebenso auch in der Zukunf, in einer großen Gesamtvorstellung zu vereinigen, wie einst der große bürgerliche Naturrechtslehrer Grotius in seinen drei Büchern * de jure beili et pacisa und wie in noch vollkommener Weise 200 Jahre später der letzte bürgerliche Philosoph Hegel seinem "Grundriß des Naturrechrs und der Staatswissenschaț (der sogenannten »Rechtsphilosophie «) die Totalität des Werdens und Seins der bürgerlichen Gesellschatt in einem groBen Gesamtbegriff entwickelt hat.

Das Arbeitsrecht tritt dem alten, positiven, erstarrten und von ihr selbst nur noch als historisch und positiv betraciteten Recht der zerfallenen bürgerlichen Gesellschaft und des zerfallenden bürgerlichen Staates scion innerhalb dieser Gesellschaft und dieses Staates selbst als das neue proletarische Recht der Arbeiterklasse gegenüber, ganz ebenso wie einst im klassischen Naturrecht von Grotius 
bis Hegel dem positiv erstarren Rechre der verfallenden feudalen Gesellsciatt das neue bürgerliche Recht des dritten Sundes gegenüber getreten ist, das später durch siegreiche revolutionäre Gewalt an die Stelle des zerbrochenen mittelalterlichen Rechts gesetzt werden sollte. Wenn also im Mittelpunkt aller unserer sozialen und sogar auch unserer politischen, innerpolitischen und außenpolitischen Kämpfe hcute tatsächlich der Kampf um die Arbeitsbedingungen der für ihre Emanzipation kämpfenden proletarischen Lohnarbeiterklasse besteht, so muß unvermeidlich auch die Theorie des modernen Arbeitsrechts in die Qual diescs Kampfes mit hineingezogen werden. In dem praktischen und theorerischen Kampf um die Schaffung und Weiterentwidklung des Arbeitsrechts « kämpft die proletarische Klasse um die Etablierung ibres Naturrechts, und dieses Arbeitsreche entaltet deshalb seinen vollen Sinn auch erst für denjenigen, der es nicht als ein fertiges Normensystem ansieht, das von einer über den kämpfenden Parteien stehenden Macht über sie gesetzt wäre, sondern es vielmehr mnaturrechtlich * begreift als ein mitten im Prozeß seines Werdens befindliches, fortwährend sich änderndes und entwickelndes Recht des Krieges und Friedens für die einander in der heurigen bürgerlichen Gesellschaft gegensätzlich gegenüberstehenden Klassen. Ein Recht des Kriegs und des Friedens für die einander in der bürgerlichen Gesellschaft gegenüberstehenden Klassen! Dieser Satz muß in seinem vollständigen Sinn begriffen werden. Dazu gehört vor allem, daß man mit den klassischen Naturrechtslehrern, mit Grotius, Hegel und Clausewitz und mit den politischen Okonomen der proletarischen Klasse, mit Marx und Lenin, das wirkliche Wesen des Krieges und dessen wahres Verhältnis zum Frieden begreił. Fast ebenso falsch wie die Abstraktion, welche das moderne Arbeitsrecht wesentlich als Friedensnorm ansieht, fast ebenso fehlerhaft wäre auch noch die andere Abstraktion, welche $z$ wischen Krieg und Frieden einen unüberbrückbaren Abgrund aufreißt und dadurch zu der Annahme gelangt, daß zwischen demjenigen Arbeitsrecht, welches die Arbeitsverhältnisse im Zustand des wirtschaftlichen Friedens beherrscht und dem Rechte der Arbeitskämpre ein grundsätzlicier Gegensatz bestände. Krieg und Frieden bilden ein Ganzes des gesellschaftlichen Lebens, so lehren uns Grotius, Kant, Fichte, Hegel und Clausewitz. Der Krieg steht dem Frieden nicht als etwas ganz und gar von ihm Verschiedenes, ihm absolut Gegensätzliches gegenüber. Sondern im Krieg ist noch der Frieden enthalten, wie im Frieden schon der Krieg enthalten war und umgekehrt: im Krieg ist schon der Friede, wie im Frieden, z. B. im Versailler Frieden, noch der Krieg enthalten ist. Man muß sich, wie Clausewitz sagt, von der gewöhnlichen Vorstellung völlig loslösen, welche meint, daß der Krieg zwar durch den politischen Verkehr der Staaten hervorgerufen würde, daß aber nun mit dern Eintritt des Kriegszustandes »jener Verkehr authöre und ein ganz anderer Zustand eintrete, welcher nur seinen eigenen Gesetzen unterworfen sei . Der Krieg ist vielmehr, nach dem bekanntesten Ausspruch von Clausewitz, mnichts als die fortgesetzte Staztspolitik mit andern Mittein. « Und ebenso ist auch der Arbeitskampf der streikenden Proletarier weiter nichts als die Fortsetzung der Bewegung, die sie zuvor mit anderen Mitteln für die gleichen wirschaftlichen und politischen Ziele geführt haben und die sie für die gleichen, für gesteigerte oder auch für herabgesetzte Ziele, auch nach der Streikbeendigung, nach der Wiederherstellung des sogenannten *Wirtschaftsfriedens", mit friedlichen und kriegerischen Mittein weiterführen werden, bis sie ihr wirkliches Klassenziel erreicht haben, bis die Arbeiterklasse gleich inrem Vorgänger, dem >dritten Stand der bürgerlichen Revolution, aus dem gesellschaflidien Nichts das gesellschafiliche Alles geworden ist. Bis dahin bleibt das Arbeitsrecht ein Recht des Krieges und des Friedens der 
in unversöhniichem, auf die Dauer unausgleichbarem Gegensatz sich gegenüberstehenden Klassen der bürgerlicten Gesellschaft.

Es gibt in der allgemeinen philosophischen Begriffswelt der Philosophie des deutschen Idealismus, besonders bei dem Philosophen Fichte, und ebenso auch in der speziellen Kriegsphilosophie des Fidteaners Clausewitz einen für den heutigen Leser zunächst etwas seltsamen Begriff: den Begriff des absoluten oder heiligen Krieges, an dessen Wesen zwar auch jeder gewöhnliche Krieg der wirklichen Gesćichte in geringerem oder größerem Maße teilhat, der aber voll entwickelt erst in der damaligen Gegenwart in die Erscheinung getreten sein soll: in den Kriegen der $\mathrm{I} 4$ französischen Revolutionsarmeen, in den Schlachten des großen $\mathrm{Na}$ poleon, der von den deutschen Philosophen als * der Kriegsgott selber « bezeicnnet wird, und vielleicht auch noch in den durch diese napoleonischen Kriege ausgelösten und an ihrem Muster entwickelten Gegenkriegen, z. B. dem deutschen Befreiungskrieg $18 \times 3$. Dieser *absolute « ist vor den gewöhnlichen Kriegen ausgezeichnet vor allem dadurch, daß sein $Z$ wedk niche in der Erringung eines bloBen Vorteils über den Gegner besteht, sondern in seiner völligen Niederẅerfung; » sei es, da B man ihn politisch vernichten oder bloß wehrlos machen und also zu jedem beliebigen Frieden $z$ wingen will $\alpha$. Ob ein solcher absoluter Krieg geführt wird, das hängt nicht allein von dem Willen der Kriegsführenden ab sondern hauptsächlich von den Energien derjenigen Politik, die in einem solchen Kriege ihre Ziele verfolgt und die ihrerseits wiederum nicht die Sache des freien Willensenrschlusses von einzelnen Staatsmännern ist sondern inre eigentlichen Wurzeln in der gesamten und besonders der wirtschafilichen Entwicklung der betreffenden Gesellscíaft hat.

Auf den Krieg der Stâaten bezogen scheint dieser Fichte-Clausewitzsche Begriff des *heiligen " oder nabsoluten K Krieges eine gewisse Mystik an sich zu haben. Wir haben in den Tagen ron $19 \mathrm{I} 4$ die schauderhafteste Karrikatur eines solchen sheiligen Krieges« erlebt und stehen dem ganzen Begriff des heiligen Krieges mit theoretisciem und praktischem Mißtrauen gegenüber. Aber gerade wenn wir diese Unterscieidung der gewöhnlichen Kriege und des absoluten Krieges jetzt auci auf unser Gebies, auf das Gebier der Arbeitskämpie der proletarischen Klasse übertragen, verliert der Begriff des absoluten Krieges in dieser Anwendung jegliche Mystik, - und er vermag uns dann sogar auch noch rückwirkend jenes dunkle Rä̈tsel rationell auflösen, das die klassischen bürgerlichen Philosophen in mystischer Form mit ihrem Begriff eines über alie gewöhnlichen Kriege weit hinausragenden heiligen « Krieges zu lösen versucht haben.

Wir haben schon vorhin betont, daß wir das wirkliche Wesen eines noci so kleinen und unbedeurenden Lohnkampfes oder sonstigen Arbeitskampfes ers: dann voll begreifen, wenn wir diesen einze!nen Arbeirskampf nicht als isoliertes Phänomen für siç berraciten sondern als einen bloßen Teilvorgang in den ungeheuren, letzten Endes auf die Eroberung der politischen Macht für die Arbeiterklasse abzielenden Gesamtprozeß der proletarischen Revolution. In all diesen mannigfacien kleinen und großen, vereinzelten und massenhaften, wirtschaftlichen, sozialen, politischen und militärischen Kämpîen und in den "friedlichen « Aktionen, welche zwischen diesen eigentlichen Kämpfen liegen, arbeitet und kämoft sich also die Arbeiterklasse endlich heran zu jenem letzten und entscheidenden Endkampfe, der um die Eroberung der politischen Macht selber geführt wird. Dieser Kampf ist dann der *absolute « Krieg, den das Proletariat in seiner gescichutichen Stunde zu führen berufen ist, ein Krieg, der nicht mehr mit der Erringung eines Einzelvorteils über den Gegner sondern nur noch mit der vollständigen Niederwerfung des Gegeners sein politisches Ziel erreichen kann. 
Diejenige Rolle aber, die dieser vollbewuBte Endkampf - mblutiger Tod oder das Nichts, so ist die Frage unerbittlich gestelle! - für das revolutionäre Proletariat zu spielen hat, dieselbe Rolle haben in früheren Jahrhunderten jene Kriege gespielt, durch die ein Napoleon *jenseits der französischen Grenzen überall die feudalen Gestaltungen wegfegte, soweit es nörig war, um der bürgerlichen Gesellschaft in Frankreich eine entsprechende zeirgenössische Umgebung auf dem europäischen Kontinent zu verschaffen*, - und ebenso in höherem und niedrigerem Grade auch alle sogenannten *Völkerkriege * der bürgerlichen revolutionären Epoche, die auf die Konstituierung der bürgerlichen Nationalstaaten abzielten, sie waren objektiv notwendige Teil jenes Endkampfes, durch den der dritre Stand, die bürgerlicie Klasse, allenthalben in Europa die ganze Macht über Staat und Gesellschaft für sich erobert und die Welt nach seinem Bilde umgestalter hat. Aus dieser Quelle entsprang ihnen jene unerhörte Energie und Rücksichtslosigkeit der Kriegführung, die den Schrecken und die staunende Begeisterung ihrer Mitwelt so häufig erregt hat. Aber eines unterscheidet doch diese vergangenen Machthämpfe der bürgerlichen Klasse von dem gegenwärtigen und zuhünftgen Machtkampf der proletarischen Klasse, - das gleiche, was auch den Naturrechtsbegriff der bürgerlichen Klassiker von jener neuen »naturrechelichen « Auffassung unterscheidet, die heute das revolutionäre Proletariat dem begriffslosen Positivismus der bürgerlichen Jurisprudenz entgegenstellt. Die Helden und Akteurs jener blutigen Kämpfe der bürgerlichen Heroenzeit kämptten teils mit einem einfachen mystiscien Glauben an die Heiligkeit ihrer Aufgabe, wie Crompell, teils mit dem Bewußtsein, die ewigen Menschenrechte zu verwirklichen, wie Robespierre und St. Juse. Sie wußten wenig von der wirklichen geschichtlichen Bedeutung der Kämpfe, die sie auszuführen hatten. So wenig wie die klassischen Naturrechtslehrer davon wußten, daß die von ihnen theoretisch eroberten heiligen und ewigen Geburtsrechte der Menschheit nur den der Produktionsweise einer bestimmen geschichrlichen Epoche angepaßten Rechtszustand der bürgerlichen Klassengesellschaft zum Ausdrudk brachten. Das Proletariat ist die erste Klasse, die mit einem vollständigen und klaren Bewußtsein ihre weltgeschichtliche Aufgabe zu erfüllen unternimmt. Es will das Naturrecht seiner Klasse, um dessen Verwirklichung es seine heurigen Kämpfe führt, nach seinem Siege nicht verewigen, sondern mit den Klassen und Klassengegensätzen zugleich auch das Reche der Arbeiterklasse aufheben. In der proletarischen Revolution des 19. und 20. Jahrhunderts ist die Revolution endlich bei ihrem eigenen Inhalt angekommen.

\section{Anmerkung zu Korschs Rechtstheorie}

Karl Korsch ist ein Jurist, dessen Beitrag zur Recitstheorie von der Rechtswissenschaft bisher nicht zur Kenntnis genommen worden ist. Durch die Publikation der bisher unveröffentlichten Antrittsvorlesung, die Karl Korsch am 9. Mai I92 $3^{1}$ als ordentlicher Professor an der Universitüt Jena hielt, will die Kritische Justiz beitragen zur Rezeprion der für eine materialistische Rechtscheorie with-

1 In der von Hedda Korsch 1962 erstellten Bibliographie wird als Datum des Voriesung der 9. Mai $192+$ genanne. 
tigen Schriften Korschs, die durch die Neuveröffentlichung von Korschs Besprechung der Bücher von Eugen Pašukanis und Karl Renner ${ }^{2}$ cingeleitet worden ist.

Karl Korsch wurde i $8 \$ 6$ in Tostede bei Hamburg geboren; I9I I juristische Dissertation mit der Arbeit •Die Beweislast beim qualifizierten Geständnis «; 19121914 Studium in England, Sympathien für die Fabian Society; 1914-1918 Frontoffizier (trug, wie es heißt, auch bei gefährlichen Unternehmen nie eine Waffe); Kriegsende Eintritt in die USPD, später Ubertritt mit der Mchrheit der LSPD in die KPD (obwohl Gegner dieses Zusammenschlusses); 1919 Privatdozent in Jena; 1923 Justizminister der sozialdemokratisch-kommunistischen Koalitionsregierung in Thüringen, Ordinarius in Jena (muß sich in der Folgezeit mit Lehrverbot auscinandersetzen, setzt in einem Rechtsstrcit scin Recht durch, verzichtet in einer Vercinbarung jedoch darauf, an der Universität Jena zu lehren und lebe von 1924-1933 in Berlin); 1924-1928 Mitglied des Reichstages; 1926 Ausschluß aus der KPD; nach I 933 Emigration; $1935-1936$ besonders enge $\mathrm{Zu}$ sammenarbeit mit Bert Brecht in Dänemark; ab 1936 in den USA; $196 \mathrm{t}$ dort gestorben.

Korsch hat es selbst nicht für sinnvoll gehalten, den Text der Vorlesung * Jus belli ac pacis im Arbeitsrecht « zu publizieren. Vermutlich deshalb, weil er eine Veröffentliciung im bürgerlichen Wissenschaftsberrieb für sinnlos hielt und das, was er den yom Arbeitsrechr Betrofienen zu sagen hatte, in seinem Arbeitsrecht für Betriebsräte ${ }^{3}$ bereits vorgelegt hatte. Den rechtstheoretisch interessanten Teil dieser Arbeit ( $(5)$ ) hatte er unter der Uberschrift "Wesen der A.rbeitsverfassung nach bürgerlichem und nach sozialem Recht « in der Zeitschrift Arbeitsrecht (Jg. 9, $19: 2$, S. 614 f. .) veröffentlicht. Der Vorlesungstext führt rechtstheoretisch nur wenig über die dort niedergelegte Position hinaus. Er bringt jedoch die waktivistische Komponente", die für Korschs gesamtes theoretisches Werk bestimmend ist, besonders prägnant zum Ausdruck. Auch wenn wir nach den Erfahrungen mit dem Faschismus Klassenauseinandersetzungen nicht mehr stets und unter allen Umständen vorbehaltlos auf die Zuspitzung in einen *absoluten Krieg * zutreiben können, bleibt es wichtig, an diese aktivistische Komponente zu erinnern, nicht zuletze deshalb, weil die entsprechenden Passagen in der Neuausgabe von Arbeitsrecht fïr Betriebsräte im Jahre 1968 fehlen.

Korsch har im Arbeitsrecht für Betriebsräte von den für eine "Übergangsepoche charakteristischen Kämpfen zwischen der rein bürgerlichrechtlichen und sozialrecticlichen Auffassung des Arbeitsrecits « gesprochen und davon, daß der Kampf für eine ssozialrechrticie Auffassung der Arbeitsverhälrnisse" durch den poiitischen Kampf der "Angenörigen der Arbeiterklasse defensiv und of̈ensiv zu führen ist: "Dejensiv, indem sie von der jeweils errungenen Position nicht eine Haaresbreite ohne Kampf preisgeben. Offensiv, indem sie von jeder gerwonnenen Position aus unablässig weiter vorwärtsdringen, bis sie in Gemeinschaft der Masse ihrer Mitkämpfer die fcindliche Festung endlich wirklich eroberr haben. « Daneben hält Korsch die »juristische Akrion « für wichtig, die diesen Kampf unterstützt und die für diesen Kampf »theoretisch ausrüstet«: »In einer

: Karl Korsch, Literarurberichta [über Renner und Pašukanis], in: Archiv fär die Gesdrónte des Sozalismus und der Arbcuterbewegung. Jg. Is, 2930, S. 308; Neudruds in: Eugen Pasukanis, Allgemeine Rechislcime und Marxismus, Frankfurt am Main, 1966, Vorworr; Karl Korsch, Die materiaisstisdie Gescrichesauffassung und andere Sohrifien, hisgg. von Ërici Gerlaci, Frankiurs an Main, 1971 , S. 157 fr.

3 Karl Korsch, Arbeitsrecit für Betriebsräse, Betlin, 19:2; verkürztc Neuautlage, Fran'ifirs am Marn Wien, r968. Besprocien von L. Unterseher in $K J \mathrm{j} / 68, \mathrm{~S}$. 9 I $\mathrm{f}$.

+ Kin! Korsch, Arbcitsrecht..., 192:, S. I14 u. 107; Ausgabe I968, S. 147 u. 140. 
Epoche, wo in allen Schichten des gesellschaftlichen Lebens sich zwei Klassen feindlich gegenüberstehen, ohne daß vorläuffg eine der anderen völlig Herr zu werden, oder zu bleiben vermag, kann auch im Recht weder der alte, rein bürgerliche Standpunkt des Privatrechts unverändert aufrechterhalten werden (der das Arbeitsverhältnis als eine auf frcien Verträgen beruhende Privatangelegenheit der Beteiligren ansah), noch der neue rein proletarische Standpunkt des Sozialrechts (der das Arbeitsverhältnis als ein lediglich auf die gesellschaftiche Zusammenarbcit gegründetes Gemeinschaftsverhälnnis ansieht). Vielmehr liegen sich diese beiden Rechtsauffassungen fortwährend in den Haaren, - wie in den Homerischen Gedichten an den irdischen Kämpfen der Griechen und Trojaner um Ilions stolze Feste auch die olympischen Görter in ihrer Weise herzhaften Anteil nehmen. ${ }^{5}$ Korsch hat verhindern wollen, daß solche Sätze erneut eine - juristische Weltanschauung « begründen. So schreibt er zum Betriebsrätcgesetz: - Die größte Gefahr, die das Betriebsrätegesetz der Arbeiterklasse gebracht hat, ist die, daß auch die gesetzlichen Betriebsräte sich mit sarbeitsgemeinschaftlicheme Geiste errüllen und sich selbst als Glieder einer auf Verbrüderung von Kapital und Arbeit beruhenden sogenannten ,Gemeinwirtschaft، zu betrachten anfanfangen. ${ }^{\circ}$ Noch deutlicher wird seine Auffassung durch die Feststellung: "Auch für theoretische Sätze der sozialistischen Rechtslehre gilt der Satz des Kommunistischen Manifestes, wonach die stheoretischen Sätze der Kommunisten keine aus sich selbst heraus gültigen ewigen Wahrheiten sein wollen, sondern nur als rallgemeine Ausdrücke tatsächlicher Verhältnisse eines existierenden Klassenkampfes، zu betrachten sind. ${ }^{i}$ Diese Auffassung entspricht der im Marxismus und Philosophie ${ }^{8}$ dargelegten Auffassung: "So wenig durch die ökonomische Aktion der revolutionären Klasse die politische Aktion überflüssig gemacht wird, so wenig wird auch durch die ökonomische und polizische Aktion zusammen die geistige Aktion überflüssig gemacht $[\ldots]$ « $^{8}$ Für den Kampf um Rechtspositionen gilt, was Korsch über die »philosophische Aktion sagre: „Der philosophische Kampf der Ideen ist vom proletarischen Standpunkt aus nicht die Basis sondern nur eine vergängliche Form des revolutionären Klassenkampies, der die geschichtliche Entwicklung unserer Zeit bestimmt. «10

Für Korsch vermag nur Dialektik diese Anerkennung der juristischen Aktion mit ihrer Aufhebung in der politisch geführten sozialen Revolution zusammenzubringen. In diese Richtung weisen einzelne Passagen der Vorlesung "Jus belli ac pacis im Arbeitsrecht« ebenso wie die Scilußsätze des Arbeitsrechts für Betriebsräte (die in der Neuveröfentliciung I 968 nicht mit abgeàruckt worden sind):

-(..) bier wie in allen wirklichen Konflikten, die aufgrund des Betriebsrätegesetzes entsteber, (belfer) die gesetzlichen Mittel des, latenten Klassenkampjesr nioht mebr, und im streike bricht der ofjene Klassenkrieg aus. So laute: auct hier, in einer rechtlichen Untersuchung, das letzte Wort der sozialen Wissenschaft, daß bei einer Ordnung der Dinge, wo es noch Klassen und Klassengegensätze gibt, auch die kleinsten und unscreinbarsten Teilaufgaben der unterdrüd-

5 Ebd. S. Irs; Ausgabe 1968, S. I49 F.

SEbd., S. is s.

7 Ebd., S. IIs; Ausgabe :968, S. I 49 .

8 Karl Korsch, Marxismus und Philosophie, [zuerse 1923], Neudruds der 2. Aurilage, hrsgg. von

Erici Gerlaci, Frankfure am Main/Wien, r 966.

- Ebd., S. I3s.

10 Karl Korsch, - Lenins Philosophies, in: Living Marxism, November 1938 , S. 1;9-344; deutsch: -Zur Philosophie Leninse, in: Anton Pannekoek, Lenin als Philosoph. Frankiurt am Main/ Wien, 1969, S. 130. 
ten und zu ihrer Befreiung aufsteigenden Klasse endgültig und bis auf den Grund immer nur akf revolusionärem Wege gelüst werden können. Denn der Streike der Arbeiter in der kapttalistiscijen Gesellsdaft ist nicht nur die Vorübung zu einer irgendwann einmal zu vollbringenden Umwälzung dieser Geselischat. Er ist vielmehr, wenn audı in seiner Wirkung noch räumlich und zeitlich beschränke, innerhalb dieser Gesellschaft schon dic förmliche Aufhebung der Grundlagen dieser Gesellschaft. Das heißt: er ist selbst schon ein Teil der sozialen Revolution. $\alpha^{11}$

Karl Korsch hat diesen von ihm am Anfang der zwanziger Jahre entwickelten Ansatz, die Einteilung arbeitsrechrlicher Normen in bürgerlichrechrliche und sozialrechtliche und die Notwendigkeit "diesen ganzen Unterschied letzten Endes aufzuheben s, s: später weder konkretisiert noch weiterentwidkelt. Die Voraussetzung für eine s, Revolutionierunge des herrschenden bürgerlichen Arbeitsrechts, soweit eine solche Revolutionierung mit theoretischen Mitreln überhaupt möglich ist ${ }^{13}$, waren für Korsch schon kurze Zeit später - das zeigr die Schrift von Karl Korsch, Um die Tariffühigkeit. Eine Untersuchung ̈̈ber die hestigen. Entrvicklungstendenzen der Gewerkschaftsuewegung, Berlin I 928 - entfallen. Korsch hat den am Anfang der zwanziger Jahre entwidkelten Ansatz nicht preisgegeben sonst hätre er im Jahre 1930 in seinem Beitrag zu Renner und Pašukanis nicht den Aufsatz \& Juristensozialismus ${ }^{15}$ aus der Nesten Zeit, I887, als das "Testament von Friedrich Engels für die Beurteilung eines sozialistischen Rechtsprogramms und also auch einer sozialistischen Rechrstheorie nennen können*. ${ }^{1 B}$ Doch diese Sctrił weist keine Perspektive, sie zeigt keinen konkreten Ansatz, Rechrspositionen dialektisch aufzuheben, sic bleibr eine Abwehrschrift gegen die *Wiederanerkennung und Wiederherstellung der Recirsideologie und der darin verkleideten Wirklichkeit « ${ }^{27}$

Für eine materialistische Verfassungstheorie sind auch die Analysen von Bedeutung, die Korsch vorlegt in Auscinandersetzung einerseits mit einem verselbständigten und zum Vertassungsmodell entwidselten Räresystem und andererseits mit der Doppelfunktion des Staztes, der zum einen als entschiedenes Instrument der soziaien Reyolution dienen zum anderen auch als Mitrel der Konterrevolution gehandhabt werden kann. Korsch fragt gegen Ende der zcer Jahre:

- Wie sind Marz und Engels, die glünenden Bewunderer des vom Konvent der großen französiscien Revolution verwirklichten zentralistischen Systems de: revolutionären Diktatur dazu gekommen, als politische Form der revolutionären Dikiatur des Proletariats gerade die jenem System scheinbar entgegengeserz:e Kommuner anzusehen? «:s Korsch zeigr bei der Entfaltung dieser Fragestellung nicht nur exemplarisch, was Anwendung des Marxismus auf den Marxismus und was historische Spezifizierung heißt, er stellt damit zugleich auch die Voraussetzung derjenigen in Frage, die mglauben, daß es irgendweicie skommunales oder srätemäßiger Verfassungsformen gäbe, durch deren Einführung der von der revolutionären proletarischen Partei regierte Staat den jedem Staar annaftende Charakter eines Instruments der Klassenunterdrückung am En-

11 Karl Korsci, Arbeirsrecis: đür Be:rebsräte, 1922, S. 196.

1: Ë́c.', S. II7, Ausgioe Ig65, S. 150.

13 Ebd., Ausgabe 196S, S. I5I.

14 Die Scirirt ist ais Nacidrud erhältlich, besprocien von W. Boci in KJ $4 / 7 \mathrm{x}, \mathrm{S}+5 \mathrm{I}$ f.

15 [Friedric Engels u. Karl Kaursiy], Jurisien-Sozialismusa, MEVV, Bd. 2I, S. 491-509.

16 Kanl Korsci, a. I. O. (Anm. 2), Argiviv..., S. 617; Vorzort, S. IX; Gerizci, S. 164.

iT Ebd., Aroiso. .., S. 618; Vorwort, S. XI; Gerlaci, S. I66.

$18 \mathrm{Karl}$ Korsh, sRevolutionäre Kommunes [zuerse in: Die Aktion, 2931], in: Karl Korsci, Schrificn zisr Soz:alisicrung, hrsgö. von Erid Gerlaci, Frankfurt am Máa, 1969, S. 102. 
de gänzlich abstreifen könnte. ${ }^{19}$ Zur gleichen Zeit stellt Korsch die Frage, ob der - Stazrk wnur die organisicrte und zentralisierte Gewalt der Gesellschat, (ist), die ebenso gut von dem revolutionären Proletariat gegen das Kapital, wie von der Bourgcoisic gegen das Proletariat gehandhabt werden kann.«o Korsch hat später immer wieder die in ein Bild gepreßte Antwort wiederholt, daß die proletarische Revolutionstheorie win jeder Beziehung, im Inhalt und in der Methode behafter ist mit den Muttermalen des Jakobinismus, der bürgerlichen Revolutionstheorie. «"1 Das habe zu einer "Uberbetonung des Staates als des entscheidenden Instruments der sozialen Revolution ${ }^{22}$ geführt. Die Ausarbeitung dessen, was das konkret für die politische Praxis bedeutet, hat Korsch niche vorgelegt. Es bleibe zu prüfen, in weichem Umfang diese These bestimmt wird durch die Erfahrungen der spanischen Revolution, 23 der Entwicklung der UdSSR 24 und des faschistischen Staats. In diesem Zusammenhang sind die im Jahre 1932 in der von Harro Schulze-Boysen herausgegebenen Zeitschrift Der Gegner erschienenen "Thesen zur Kritik des faschistischen Staatsbegriffs zu beachren, in denen es heißr:

-I. r. Der faschistische Staat ist ein moderner Staat. Er bedeutet keine Rückkehr zu vorbürgerlichen Staatsstrukturen. Der Korporativstaat hat nichts zu run mit dern ,Ständestaat<. - Der Faschismus als „Gegenreform«. [...] III. I. Die faschistische Umgestaltung bedeutet keine ökonomische Revolution, keine radikale Sprengung alter Produktionsverhältnisse und Entfessclung neuer Produkrivkräfte. Hierin besteht - außer gegebenen Unterschieden der materiellen Möglichkeiten und der Größenordnung - der Hauptunterschied zwischen Faschismus und Bolschewismus. 2. Der faschistische Staat bedeutet die Vereinigung der ökonomischen und politischen Macht der Bourgeoisie gegen das Proletariat, also niche Uberwindung des Klassenstaates, sondern Herstellung des Klassenstaates in der Form des Klassenstaates. 3. Die neue Form der Verbindung der ökonomischen und politischen Klassenmache der Bourgeoisie im faschistischen totalen Staats erfordert neue Formen der Verbindung der ökonomischen und politischen Aktion des Proletariats. «25

Jürgen Seifert

10 Ebd., S. 167 ; vgl. in diesem Zusammenhang aud sRevolutionäre Kommunes [zuerst in: Die Aksion, 1929], éd., S. $g$ I fi.

30 Karl Korsch, Das Problem Stazeseinheit-Föderalismus in der Französischen Revolutiona, in: Arobu jür die Geschichze des Sazialismus und der Arbezzerbewegungr, Jg. IS. 1930. S. 140 Niacidruck in: Karl Korsch, Revolustonärer Klassenkampi, Berlin (Kollektir-Verlag, Rollbergstr. 67), 1972, S. Ss.

$"$ Rarl Korsch, , Thesen über Hegel und die Revolution, in: Der Gegner, 1932, H. 3. S. II : Ncudruds: alternatıse, H. 4 r (April 1965). S.67; ähniliche Formuiienungen finden wir in: Xarl Korsch, Karl Marx, hrsgg. von Götz Langkau, Frankfurt am Main, Wien, rg67 [zuerst: London :938], S. 204; ferner: Karl Korsch, state and Counter-Revolutions, in: The modern Quarterly, Jg. 25, 1939, H. 2, S. 66.

- Karl Korsch, so Thesen über Marxismus heute [gesdrieben 1950], in: alternative, H. 4I (April 1969), 89; als sbesonders kritische Punkte im Marxismuse kritisiert Ǩorsch u. a. das sbedingungslose Festhaleen an den politischen Formen der bürgerlichen Revolutions.

z Vgl. dazu Korschs Beiträge übe: die spanische Revolution und die Kollektivierung in Spanien in: Sörifien zur Sozialisiermng, a. a. O. (Anm. 17).

24 Vgl. dazu Karl Korsch, state an Counter-Revolutione, a. a. O. (Anm. 20), S.69: whow did is happen that the workers' state emerging from the 1917 revolution in Russia was slowly and without any ,Thermidors or ,Brumaires transiomed from an instrument of the proletarian Revolution into an instrument of the present-day European counter-revolution? e

es Karl Korsch, - Thesen zur Kritik des faschistischen Srazesbegriffese, in: Der Gegner, 1932, H. $4 / 5$, S. 20 f. 